\title{
The evaluation of radiological measurements used in diagnosis and treatment of syndesmosis injury: A regional study in Turkey
}

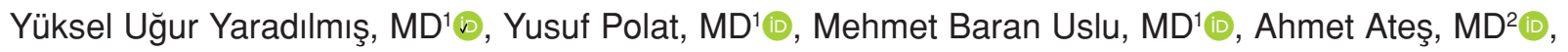 \\ İsmail Demirkale, $\mathrm{MD}^{2} \mathbb{C}$, Murat Altay, $\mathrm{MD}^{2} \odot$ \\ 'Department of Orthopaedics and Traumatology, Çankırı State Hospital, Çankııı, Turkey \\ 2Department of Orthopaedics and Traumatology, University of Health Sciences, Keçiören Health Practice and Research Center, Ankara, Turkey
}

Syndesmosis is a ligament complex composed of the anterior inferior tibiofibular ligament, interosseous ligament and posterior inferior tibiofibular ligament. Syndesmosis is the essential component of distal tibiofibular joint. Pain and loss of ankle function are unavoidable in cases of syndesmosis injuries. ${ }^{[1,2]}$ Early diagnosis and selection of the appropriate treatment method are important since overlooking or improper treatment of the injury will cause negative functional outcomes in the patient. ${ }^{[3]}$

Many methods have been defined for the diagnosis of syndesmosis. In the diagnosis process of syndesmosis injuries, computed tomography (CT) is sensitive and specific in evaluation of the osseous pathologies and assessment of the rotational alignment of syndesmosis, while magnetic resonance imaging (MRI) has sensitivity and specificity in evaluation of the ligaments. ${ }^{[4,5]}$ The findings of physical examination (squeeze test, external rotation test) and direct radiographs are used in the first evaluation of a syndesmosis injury. Approximately

Received: September 02, 2019

Accepted: November 14, 2019

Published online: March 02, 2020

Correspondence: Yüksel Uğur Yaradılmış, MD. Kırıkevler Mah., No: 236/1, 18100 Çankırı, Türkiye.

E-mail: ugur_yaradilmis@outlook.com

Doi: $10.5606 /$ ehc. 2020.71132

Citation: Yaradılmıș YU, Polat Y, Uslu MB, Ates A, Demirkale I, Altay $M$. The evaluation of radiological measurements used in diagnosis and treatment of syndesmosis injury: A regional study in Turkey. J† Dis Relat Surg 2020;31(1):123-129.

\section{ABSTRACT}

Objectives: This study aims to improve the diagnostic reliability of syndesmosis injuries through evaluation of radiological measurements in the Turkish population and to provide mean reference values to prevent malreduction and overcompression during the treatment.

Patients and methods: This retrospective study was performed between January 2018 and May 2018. The bilateral anteroposterior (AP) and lateral radiographs of 100 patients (60 males, 40 females; mean age 42.9 years; range, 23 to 72 years) who presented at our polyclinic were analyzed. Tibiofibular overlap (TFO), tibiofibular clear space (TFCS) and medial clear space (MCS) measurements were performed on the AP radiographs. The lateral radiographs were evaluated in respect of the anterior tibiofibular interval (ATFI), posterior tibiofibular interval (PTFI), and anterior tibiofibular ratio (ATFR) for syndesmosis reduction assessment. The lower and upper limits, mean and median values of the measurements were recorded.

Results: The measurements on the AP radiographs were determined to be as TFO: $7.9 \pm 2.4 \mathrm{~mm}$ (4-13), TFCS: $3.8 \pm 0.9 \mathrm{~mm}$ (2.2-6), MCS: $3.3 \pm 0.4 \mathrm{~mm}(2.7-4.5)$, and superior clear space: $3.3 \pm 0.3 \mathrm{~mm}(2.7-3.8)$. According to the measurements on the AP radiographs, the TFCS did not show any difference in terms of the variables of age, gender and side $(p=0.070, p=0.219$ and $p=1.0$, respectively). These measurements on the AP radiographs showed a high statistical consistency in terms of side $(\mathrm{p}=0.72, \mathrm{p}=1.0, \mathrm{p}=0.900$ and $\mathrm{p}=0.920$, respectively). The measurements on the lateral radiographs were as ATFI: $12.8 \pm 2.4 \mathrm{~mm}$ (8-18), PTFI: $6.1 \pm 2.9 \mathrm{~mm}(3-15)$ and ATFR: $0.4 \pm 0.1$ (0.28-0.5). According to the measurements on the lateral radiographs, the ATFR did not show any difference in terms of the variables of age, gender and side ( $\mathrm{p}=0.750, \mathrm{p}=0.570$ and $\mathrm{p}=0.848$, respectively). The lateral measurements indicated statistical consistency in terms of side ( $\mathrm{p}=0.400, \mathrm{p}=0.260$ and $\mathrm{p}=0.848$, respectively).

Conclusion: On the AP radiographs, TFCS was found to be reliable and the intraoperative evaluation of its high consistency with the opposite extremity is appropriate to avoid overcompression. The evaluation of ATFR on lateral radiographs was found to be reliable and evaluation is recommended to avoid intraoperative malreduction.

Keywords: Malreduction, overcompression, radiological diagnosis, syndesmosis injury. 
$100 \%$ of syndesmosis injuries can be detected by MRI, although there is no consensus on performing MRI in routine practice and syndesmotic instability is essential. ${ }^{[6]}$ The accepted limit values for syndesmotic instability by direct radiographs are tibiofibular overlap (TFO) $<6 \mathrm{~mm}$, tibiofibular clear space (TFCS) $>6 \mathrm{~mm}$ and medial clear space (MCS) $>4 \mathrm{~mm}$ on anteroposterior (AP) radiographs. ${ }^{[7]}$ It is possible that radiographic data may vary depending on personal variables and the surgeon may fail to take these into consideration. In a review by Prakash, ${ }^{[8]}$ it was reported that radiological results may vary depending on age and gender.

The selection of the appropriate treatment method is important together with accurate diagnosis. At the present time, diagnostic methods are prominently discussed and reduction assessment may be kept in the background. However, anatomic reduction is necessary for successful outcomes, as even $1.5 \mathrm{~mm}$ malreduction may cause negative clinical outcomes. ${ }^{[3,9]}$ Gardner et al..$^{[10]}$ detected a malreduction rate reaching $50 \%$ in the postoperative CT evaluations. The amount of syndesmosis needed to be compressed for a reduced syndesmosis should be determined because overcompression of syndesmosis may affect the movement and functional outcomes of the ankle. ${ }^{[11]}$

In this study, we aimed to improve the diagnostic reliability of syndesmosis injuries through evaluation of radiological measurements in the Turkish population and to provide mean reference values to prevent malreduction and overcompression during the treatment.

\section{PATIENTS AND METHODS}

Analysis was performed of the radiographs of patients who presented at Çankırı State Hospital Orthopaedics and Traumatology Department with foot complaints without trauma between January 2018 and May 2018. Direct radiographs were retrospectively obtained from the Picture Archiving and Communication System of our hospital. The patients included in the study were aged between 22-75 years and had standard direct radiographs. The patients without standard radiographs, or with a congenital deformity or surgical history were excluded. Thus, a total of 100 patients ( 60 males, 40 females; mean age 42.9 years; range, 23 to 72 years) were included. The radiological measurements of the 200 ankles were performed by three orthopedics and traumatology specialists. The age, gender and side of the patients were recorded. Tibiofibular overlap, TFCS and MCS measurements were performed on the AP radiographs. ${ }^{[12]}$ The patient measurements were evaluated using the anterior tibiofibular ratio (ATFR), anterior tibiofibular interval (ATFI) and posterior tibiofibular interval (PTFI) on the lateral radiographs. ${ }^{[13]}$ Rotation of the radiographs was determined by evaluating the overlap of the talar dome. Mean and median values, lower and upper limits were calculated from the measurements on the AP and lateral radiographs, and were compared with the cut-off values accepted in the literature. The variability of the radiological measurements was analyzed according to age, gender and side. The study protocol was approved by the Çankırı State Hospital Ethics Committee and Çankırı Provincial Health Directorate (Date: 08.07.2019, No: 108). A written informed consent was obtained from each patient. The study was conducted in accordance with the principles of the Declaration of Helsinki.

On the AP radiographs, the measurements were taken of TFO: the maximum distance that the anterior tibial tubercle and fibula overlap in a distance of $1 \mathrm{~cm}$ from the tibial plafond, the TFCS: the distance between the tibia and fibula in a distance of $1 \mathrm{~cm}$ from the tibial plafond, the MCS and superior clear space (SCS): the distance between the distal tibial and talus (Figure 1).

On the lateral radiographs, the measurements were taken of the ATFR: the ratio of the distance between the anterior cortex of the tibia and the anterior cortex of the fibula to the distance between the anterior tibial cortex and the posterior tibial cortex, the ATFI: the distance between the anterior tibial cortex and the anterior fibular cortex, and the PTFI: the distance between the posterior tibial cortex and the posterior fibular cortex (Figure 2).

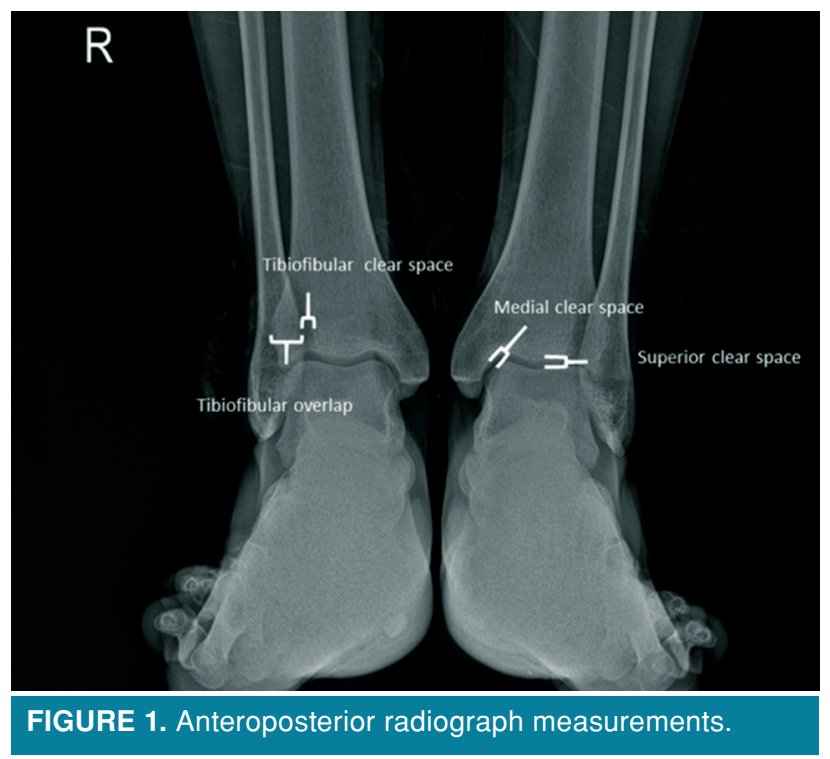




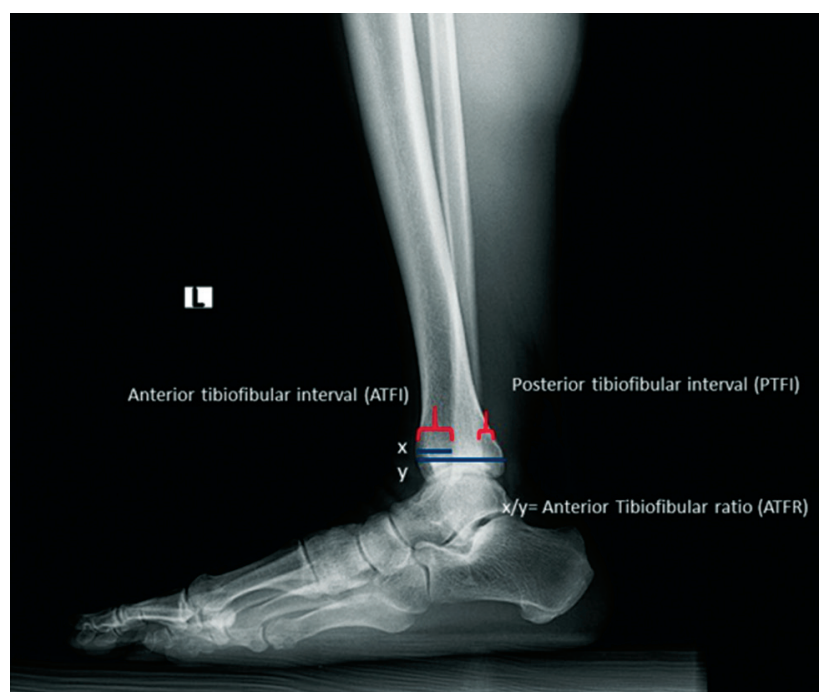

FIGURE 2. Lateral radiograph measurements.

\section{Statistical analysis}

Data analysis was performed using the IBM SPSS version 22.0 software (IBM Corp., Armonk, NY, USA) with a confidence interval of $95 \%$. In the statistical analysis, frequency distribution was used for qualitative variables while minimum, maximum and mean values were given for quantitative variables. The Shapiro-Wilk test indicated no abnormality of data distribution. The Mann-Whitney $U$ test and the Kruskal-Wallis test were performed. Mean values and standard deviations were calculated in the measurements (TFO, TFCS, MCS and SCS measurements, ATFR, ATFI and PTFI), and the results were evaluated in terms of age and gender using the Mann-Whitney $\mathrm{U}$ test. A $p$ value of $<0.05$ was accepted as statistically significant.

\section{RESULTS}

Bilateral measurements were taken of all the patients $(\mathrm{R} / \mathrm{L}=1 / 1)$. According to the AP radiographs, the TFO, TFCS, MCS and SCS measurements were found to be $8.0 \pm 2.4 \mathrm{~mm}(4-13$, median $=9 \mathrm{~mm}), 3.8 \pm 0.9 \mathrm{~mm}(2.2-6$, median $=4 \mathrm{~mm}), 3.3 \pm 0.4 \mathrm{~mm}(2.7-4.5$, median $=3.3 \mathrm{~mm})$ and $3.3 \pm 0.3 \mathrm{~mm}(2.7-3.8$, median $=3.3 \mathrm{~mm})$ in terms of mean \pm standard deviation and limit values, respectively (Figure 3).

The analysis of the AP radiograph measurements revealed a statistically significant difference between the genders in respect of TFO, MCS and SCS values $(\mathrm{p}=0.03, \mathrm{p}=0.02$ and $\mathrm{p}=0.01$, respectively). No statistically significant difference was determined between the genders in terms of TFCS $(p=0.219)$. In

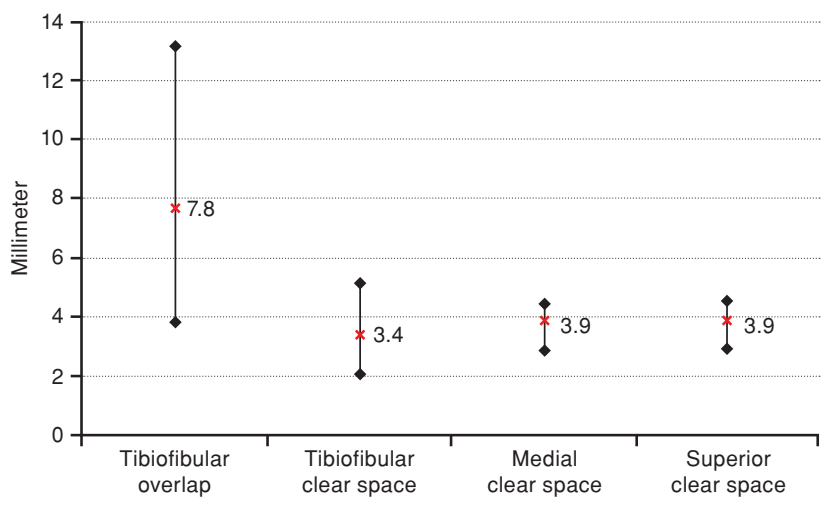

FIGURE 3. Radiological measurement on anteroposterior radiographs- minimum, maximum and mean.

females, TFO, TFCS, MCS and SCS were measured as $7.0 \pm 2.1 \mathrm{~mm}(4-9.8), 3.5 \pm 0.8 \mathrm{~mm}(2.2-4), 3.2 \pm 0.3 \mathrm{~mm}$ (2.7-3.7), and $3.3 \pm 0.2 \mathrm{~mm}$ (2.7-3.4), respectively, with lower values determined of TFO, MCS and SCS than in males whereas the TFCS was similar. According to the analysis of the AP radiograph measurements, a statistically significant difference was found between the age groups in respect of TFO $(p=0.01)$, MCS $(p=0.02)$, and SCS $(p=0.03)$, and no statistically significant difference was found in TFCS $(p=0.07)$. In the $40-60$ years age group, the TFO: 7.0 $\pm 2.1 \mathrm{~mm}$ (4-9.8), MCS: $3.2 \pm 0.3 \mathrm{~mm}$ (2.7-3.7) and SCS: $3.3 \pm 0.2 \mathrm{~mm}$ (2.7-3.4) values were found to be lower than those of the other age groups, and the TFCS: $3.5 \pm 0.8 \mathrm{~mm}(2.2-4)$ measurements were similar. The AP radiograph measurements did not indicate any correlation with increased age. The analysis of sides showed high statistical consistency; the TFO, TFCS, MCS and SCS values of the right and left sides were found to be parallel $(p=0.72, p=1.0, p=0.90$ and $p=0.92$, respectively). The AP radiograph measurements and the correlations between gender, age and side are presented in Table I.

The mean and limit values of the lateral radiograph measurements for ATFI, PTFI and ATFR were determined to be $12.8 \pm 2.4 \mathrm{~mm}(8-18$, median=10 $\mathrm{mm}), 6.1 \pm 2.9 \mathrm{~mm}(3-15$, median=8 $\mathrm{mm})$ and $0.4 \pm 0.1(0.28-0.5$, median $=0.4)$, respectively.

The analysis of the lateral radiograph measurements in respect of gender indicated no statistically significant difference between the ATFI, PTFI and ATFR values $(\mathrm{p}=0.77, \mathrm{p}=0.80$ and $\mathrm{p}=0.57$, respectively). The analysis of the lateral radiograph measurements in respect of age indicated a statistically significant difference between the ATFI and PTFI values $(\mathrm{p}=0.01$ and $\mathrm{p}=0.02$, respectively) 


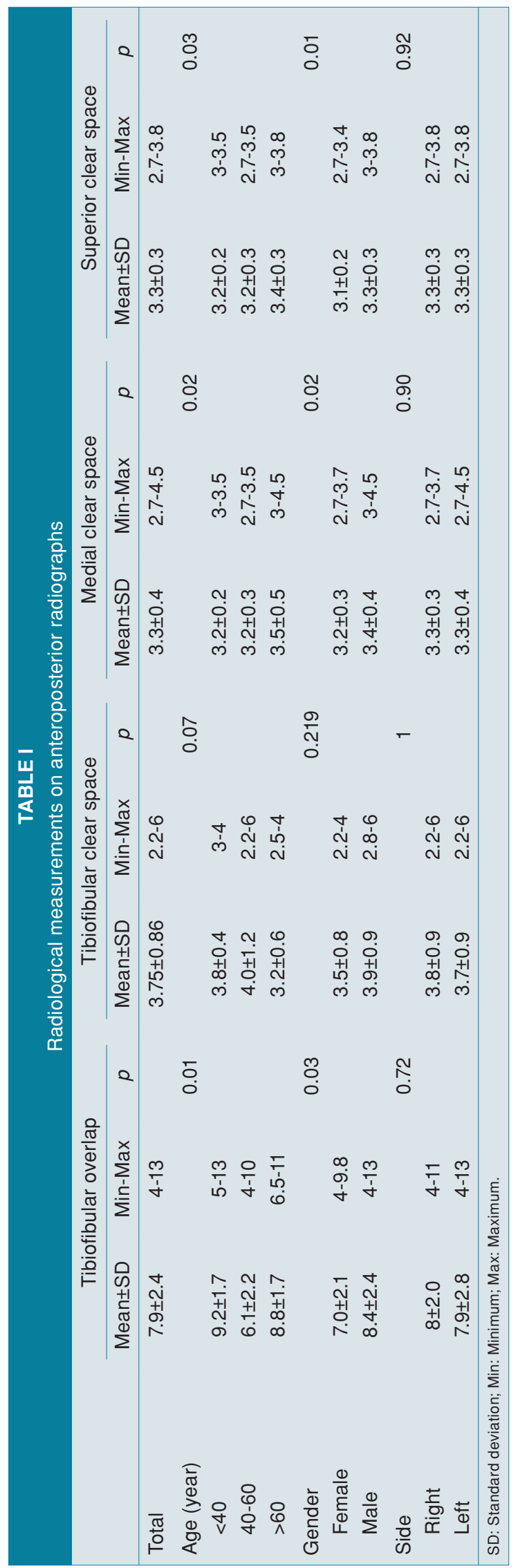

and no statistically significant difference was found between the age groups in the ATFR value ( $p=0.753$ ). The change in the ATFI and PTFI values was not correlated with increased age. The analysis of the lateral radiograph measurements in respect of side indicated no statistically significant difference between the ATFI, PTFI and ATFR values $(p=0.40$, $p=0.26$ and $p=0.848$, respectively). The lateral radiograph measurements and the correlations between gender, age and side are presented in Table II.

\section{DISCUSSION}

Syndesmosis injury can be detected by physical examination tests, direct radiograph measurements, operating theatre dynamic tests or CT or MRI. The squeeze and external rotation tests are the most widely used physical examination tests, with $20 \%$ sensitivity and $84.8 \%$ specificity in the detection of syndesmosis injury compared with MRI, which has the highest diagnostic value. ${ }^{[14]}$ This ratio ranges between $50 \%$ and $65 \%$ in the comparison of radiological measurements and MRI. ${ }^{[15]}$ However, radiological measurements still remain important in both the establishment of an accurate preoperative diagnosis and the evaluation of malreduction and overcompression.

In this study, analysis was performed of the radiological measurements accepted for syndesmosis injuries and these radiological results of a Turkish population were compared with the values accepted in the literature. ${ }^{[16]}$

In 1989, Harper and Keller ${ }^{[12]}$ evaluated syndesmosis injury with AP radiographs and provided cut-off values for radiological measurements Some studies have accepted $6 \mathrm{~mm}$ as the upper limit for TFCS ${ }^{[17,18]}$ although Shah et al. ${ }^{[19]}$ measured values reaching $8 \mathrm{~mm}$. In the current study, the upper and lower limits for TFCS were found to be $6 \mathrm{~mm}$ and $2.2 \mathrm{~mm}$, respectively, while the mean value was $3.8 \pm 0.9 \mathrm{~mm}$. Overcompression of the syndesmosis screw may affect ankle movements and consequently treatment results. ${ }^{[10,11]}$ In the current study, the minimum value for overcompression was determined as $2.2 \mathrm{~mm}$. The bilateral values regarding TFCS were found to be equal ( $\mathrm{p}=1.0)$ to prevent overcompression. The most consistent method to prevent overcompression was determined to be the measurement of the TFCS of the contralateral extremity.

It has been observed that $\mathrm{TFO}$, which can be determined more easily with AP radiographs, has a wide range between lower and upper values (4-13). 


\begin{tabular}{|c|c|c|c|c|c|c|c|c|c|}
\hline \multicolumn{10}{|c|}{$\begin{array}{l}\text { TABLE II } \\
\text { easurements on lateral radiographs }\end{array}$} \\
\hline & \multicolumn{3}{|c|}{ Anterior tibiofibular interval } & \multicolumn{3}{|c|}{ Posterior tibiofibular interval } & \multicolumn{3}{|c|}{ Anterior tibiofibular ratio } \\
\hline & Mean $\pm S D$ & Min-Max & $p$ & Mean $\pm S D$ & Min-Max & $p$ & Mean \pm SD & Min-Max & $p$ \\
\hline Total & $12.8 \pm 2.4$ & $8-18$ & & $6.1 \pm 2.9$ & $3-15$ & & $0.4 \pm 0.1$ & $0.28-0.5$ & \\
\hline Age (year) & & & 0.01 & & & 0.03 & & & 0.753 \\
\hline$<40$ & $12.3 \pm 3.1$ & $8-18$ & & $6.9 \pm 2.7$ & $3-11$ & & $0.4 \pm 0.0$ & $0.3-0.5$ & \\
\hline $40-60$ & $11.2 \pm 3.0$ & $8-18$ & & $7.9 \pm 3.8$ & $3-15$ & & $0.4 \pm 0.1$ & $0.28-0.5$ & \\
\hline$>60$ & $12.8 \pm 1.7$ & $9-15$ & & $5.8 \pm 2.8$ & $4-11$ & & $0.4 \pm 0.1$ & $0.28-0.5$ & \\
\hline Gender & & & 0.77 & & & 0.80 & & & 0.57 \\
\hline Female & $11.3 \pm 1.7$ & $8-12$ & & $7.3 \pm 3.7$ & $4-15$ & & $0.4 \pm 0.1$ & $0.3-0.5$ & \\
\hline Male & $12.3 \pm 3.3$ & $8-18$ & & $6.9 \pm 3.0$ & $3-15$ & & $0.4 \pm 0.0$ & $0.28-0.5$ & \\
\hline Side & & & 0.40 & & & 0.26 & & & 0.848 \\
\hline Right & $12.3 \pm 3.2$ & $8-18$ & & $6.4 \pm 3.0$ & $3-11$ & & $0.4 \pm 0.1$ & $0.28-0.5$ & \\
\hline Left & $10.6 \pm 2.5$ & $8-15$ & & $8.6 \pm 3.4$ & $4-15$ & & $0.4 \pm 0.1$ & $0.28-0.5$ & \\
\hline
\end{tabular}

Likewise, Harper and Keller ${ }^{[12]}$ measured values below the accepted value of $6 \mathrm{~mm}$. The elusiveness of the measurements based on TFO was also determined to be high in Turkey. Shah et al. ${ }^{[19]}$ reported that TFO measurements decreased to $2 \mathrm{~mm}$. In a cadaver study, Beumer at al. ${ }^{[20]}$ demonstrated that TFO is highly affected by rotational movements and decreased to as far as $0 \mathrm{~mm}$. Herper and Keller ${ }^{[12]}$ stated that the limit was $4 \mathrm{~mm}$, with MCS of $2.9 \mathrm{~mm}(2-5)$ and SCS of $2.8 \mathrm{~mm}(2-5)$.

Malreduction of the syndesmosis has a negative effect on functional outcomes. Precise placement of the syndesmosis screw and obtaining stability improve patient outcomes. In the recent literature, the importance of iatrogenic malreduction has been mentioned and an increasing number of studies have reported malreduction rates to be higher than the estimated rate. Translational, rotational and overcompression-induced malreduction may be encountered. However, evaluation on direct radiographs may be elusive. Some studies have detected improper reductions reaching 50\% on postoperative CT. ${ }^{[10]}$ Miller et al. ${ }^{[21]}$ analyzed syndesmosis reduction and the position of the reduction clamp in a cadaver study and determined that placement of the reduction clamp at an angle of $15-30^{\circ}$ may cause external rotation and overcompression, whereas placing the clamp in the neutral anatomical axis provides a better reduction. The classical information on neutral dorsiflexion of the foot and syndesmosis reduction has been found to be irrelevant in recent studies. Similarly, it has been attempted to determine measurements by lateral radiographs in addition to the intraoperative $\mathrm{AP}$ radiographs to improve syndesmosis reduction. In the current study, lateral radiograph measurements were evaluated using the ATFI, PTFI, and ATFR.

The ATFR was measured as $0.4 \pm 0.1(0.28-0.5)$ on the lateral radiographs of the current study. Croft et al. ${ }^{[13]}$ found this ratio to be $0.4 \pm 0.1$ with a high level of similarity. A similar radiological ratio was defined as the anteroposterior tibiofibular ratio by Grenier et al. ${ }^{[22]}$ as the relationship between the remnant of the distal tibial epiphysis and fibula. Grenier et al ${ }^{[22]}$ calculated the ratio of the anterior area of the anterior fibular cortex to the posterior area of the anterior fibular cortex (anteroposterior tibiofibular ratio=APFT) and reported it to be $0.9 \pm 0.1$ (0.63-1.31).

Lateral measurements of ATFI and PTFI have been analyzed more commonly in CT imaging studies. ${ }^{[4,23]}$ In studies by Croft et al, ${ }^{[13]}$ it was reported that the ratio values were more reliable than millimetric measurements. Although the reliability of radiological measurements in CT imaging studies on sagittal planes is high, these values do not provide intraoperative direction. Dikos et al. ${ }^{[23]}$ measured ATFI as $14.6 \pm 1.8 \mathrm{~mm}(11.0-19.4)$ on CT imaging. In the current study, the ATFI was measured as $12.8 \pm 2.4 \mathrm{~mm}(8-18)$ and PTFI as $6.11 \mathrm{~mm}(3-15)$ on direct radiographs.

Variability in radiological measurements depending on demographic parameters is normal, and so the correlation with age and gender was investigated in this study. Despite the presence of 
contrary conclusions, Brown et al. ${ }^{[24]}$ stated that AP radiograph measurements change depending on age and the values accepted as normal increase together with increasing age. In the current study, no statistically significant difference was found between the TFCS values according to age and gender, whereas TFO, MCS and SCS demonstrated variability depending on age and gender. As one of the lateral radiograph measurements, the ATFR showed no change according to age and gender and high consistency was determined with the measurements of the contralateral extremity.

The strong aspect of the current study is that, to our knowledge, this is the first study to have evaluated the radiological measurements accepted for syndesmosis injuries in the literature in a Turkish population while also including the lateral radiograph measurements for malreduction similar to the other recent studies in the literature. Nevertheless, there were some limitations to this study, primarily that although conducted in Turkey, it was a regional study, not national. Moreover, the measurements were based on standard AP radiographs and mortise X-rays were not included. It was also noticed that for ideal lateral radiographic assessments, it is necessary to pay attention to the overlap of the talar dome.

In conclusion, measurement of the TFCS on AP radiographs was found to be reliable and can be used for intraoperative assessment to avoid overcompression due to the high consistency with the contralateral extremity. The ATFR measurement on lateral radiographs was also determined to be reliable, and can be recommended particularly to avoid intraoperative malreduction.

\section{Declaration of conflicting interests}

The authors declared no conflicts of interest with respect to the authorship and/or publication of this article.

\section{Funding}

The authors received no financial support for the research and/or authorship of this article.

\section{REFERENCES}

1. Sammarco GJ, Hockenbury RT. Biomechanics of the foot and ankle. In: Nordin M, Frankel VH, editors. Basic biomechanics of the musculoskeletal system. 3rd ed. Philadelphia: Lippincott Willams \& Wilkins; 2001. p. 245-9.

2. Uğurlu M, Bozkurt M, Demirkale I, Cömert A, Acar HI, Tekdemir I. Anatomy of the lateral complex of the ankle joint in relation to peroneal tendons, distal fibula and talus: a cadaveric study. Eklem Hastalik Cerrahisi 2010;21:153-8.
3. Sagi HC, Shah AR, Sanders RW. The functional consequence of syndesmotic joint malreduction at a minimum 2-year follow-up. J Orthop Trauma 2012;26:439-43.

4. Nault ML, Hébert-Davies J, Laflamme GY, Leduc S. CT scan assessment of the syndesmosis: a new reproducible method. J Orthop Trauma 2013;27:638-41.

5. Vogl TJ, Hochmuth K, Diebold T, Lubrich J, Hofmann $\mathrm{R}$, Stöckle U, et al. Magnetic resonance imaging in the diagnosis of acute injured distal tibiofibular syndesmosis. Invest Radiol 1997;32:401-9.

6. Krähenbühl N, Weinberg MW, Davidson NP, Mills MK, Hintermann B, Saltzman CL, et al. Imaging in syndesmotic injury: a systematic literature review. Skeletal Radiol 2018;47:631-48.

7. Weber BG, Simpson LA. Corrective lengthening osteotomy of the fibula. Clin Orthop Relat Res 1985;199:61-7.

8. Anand Prakash A. Syndesmotic stability: Is there a radiological normal?-A systematic review. Foot Ankle Surg 2018;24:174-84.

9. Hamoui M, Ali M, Lovas F, Bonnel F. Troubles de rotation de la malléole fibulaire après ostéosynthèse des fractures de cheville (évaluation radiologique standard et scanographique, à propos de 20 cas). Médecine et Chirurgie du Pied 2008;24:155-66

10. Gardner MJ, Graves ML, Higgins TF, Nork SE. Technical Considerations in the Treatment of Syndesmotic Injuries Associated With Ankle Fractures. J Am Acad Orthop Surg 2015;23:510-8.

11. Phisitkul P, Ebinger T, Goetz J, Vaseenon T, Marsh JL. Forceps reduction of the syndesmosis in rotational ankle fractures: a cadaveric study. J Bone Joint Surg Am 2012;94:2256-61.

12. Harper MC, Keller TS. A radiographic evaluation of the tibiofibular syndesmosis. Foot Ankle 1989;10:156-60.

13. Croft S, Furey A, Stone C, Moores C, Wilson R. Radiographic evaluation of the ankle syndesmosis. Can J Surg 2015;58:58-62.

14. Beumer A, Swierstra BA, Mulder PG. Clinical diagnosis of syndesmotic ankle instability: evaluation of stress tests behind the curtains. Acta Orthop Scand 2002;73:667-9.

15. Kellett JJ, Lovell GA, Eriksen DA, Sampson MJ. Diagnostic imaging of ankle syndesmosis injuries: A general review. J Med Imaging Radiat Oncol 2018;62:159-68.

16. Atik OŞ. Is there something new and interesting in my article? Eklem Hastalik Cerrahisi 2019;30:69.

17. Harper MC. An anatomic and radiographic investigation of the tibiofibular clear space. Foot Ankle 1993;14:455-8.

18. Pneumaticos SG, Noble PC, Chatziioannou SN, Trevino SG. The effects of rotation on radiographic evaluation of the tibiofibular syndesmosis. Foot Ankle Int 2002;23:107-11.

19. Shah AS, Kadakia AR, Tan GJ, Karadsheh MS, Wolter TD, Sabb B. Radiographic evaluation of the normal distal tibiofibular syndesmosis. Foot Ankle Int 2012;33:870-6.

20. Beumer A, van Hemert WL, Niesing R, Entius CA, Ginai AZ, Mulder PG, et al. Radiographic measurement of the distal tibiofibular syndesmosis has limited use. Clin Orthop Relat Res 2004;423:227-34.

21. Miller AN, Barei DP, Iaquinto JM, Ledoux WR, Beingessner DM. Iatrogenic syndesmosis malreduction via clamp and 
screw placement. J Orthop Trauma 2013;27:100-6.

22. Grenier S, Benoit B, Rouleau DM, Leduc S, Laflamme GY, Liew A. APTF: anteroposterior tibiofibular ratio, a new reliable measure to assess syndesmotic reduction. J Orthop Trauma 2013;27:207-11.

23. Dikos GD, Heisler J, Choplin RH, Weber TG. Normal tibiofibular relationships at the syndesmosis on axial CT imaging. J Orthop Trauma 2012;26:433-8.

24. Brown KW, Morrison WB, Schweitzer ME, Parellada JA, Nothnagel H. MRI findings associated with distal tibiofibular syndesmosis injury. AJR Am J Roentgenol 2004;182:131-6. 